\title{
A New Approach to the Dynamic RGA Analysis of Uncertain Systems
}

\author{
Miguel Castaño Arranz ${ }^{\dagger \star}$ and Wolfgang Birk $^{\dagger}$
}

\begin{abstract}
This paper deals with DRGA analysis for uncertain systems. Uncertainties in a process model can be translated into uncertainties in the DRGA which might invalidate the decision on the variable paring in decentralized control. Bounds for the uncertainties in the DRGA of a multivariable process are derived for a given nominal process model with known uncertainty region. The resulting uncertainty region for the DRGA is used to assess the validity of decisions based on the nominal DRGA. The methodology for the computation of the bounds is currently restricted to the 2-by-2 case. Beside an explanatory example, a case study on a coal injection vessel is conducted and discussed.
\end{abstract}

\section{INTRODUCTION}

$\mathbf{T}$ HE design of process control systems for complex process plants is a difficult task and mostly based on the experience of process and design engineers. A critical step in the design is the choice of manipulated and controlled variable pairs for decentralized control. Usually, these decisions are based on the indications of variable pairing methods.

One of the first methods that was proposed in the late 1960s is the relative gain array (RGA) and its analysis, [1]. It has been first introduced as an interaction measure between the different channels in a multivariable process; but it has also been proved to be useful for other analysis, like stability, robustness, or decentralized integral controllability. Since then, many new methods and algorithms combining several methods were suggested, like partial relative gains [2], the $\mu$-interaction measure [3] and gramian based measures [4], [5], [6]. Common to all of them is the need for a multivariable process model. Moreover, most methods require linear time invariant models. These models are then used for the subsequent design of the individual controllers.

Clearly, all process models are affected by uncertainties as simplifications and approximations are unavoidable during modeling. Although control design methods are available that consider uncertainties there are currently no control structure design methods that make use of uncertainty descriptions. Thus, the validity of the decisions based on control structure methods can not be assessed. Only recently, the effect of uncertainties on control structure designed methods has received increasingly attention, i.e. the sensitivity of the RGA to uncertainties.

Uncertainties mean that there is a set of process models that represent the real process in different conditions and the

\footnotetext{
* Corresponding author: miguel. castano@ltu.se.

$\dagger$ Control Engineering Group, Department of Computer Science and Electrical Engineering, Luleå University of Technology, SE-971 87 Luleå, Sweden. Funding provided by Swedish Governmental Agency for Innovation Systems (VINNOVA) is gratefully acknowledged.
}

nominal plant is one of them. Thus, there is a set of RGAs instead of only a nominal one.

Dan Chen and Dale E. Seborg in [7] proposed an statistical description of the possible bounds of the values of the RGA due to uncertainties. In this approach, the variance and covariance between the gains of the different elements of the plant are supposed to be known, and the bounds of the static RGA for three different squared systems are analyzed and given with a confidence coefficient.

Kariwala and Skogestad derived in [8] a method for obtaining the maximum possible magnitude of the values of the RGA due to uncertainties. Although the description of uncertainties used for this analysis is assumed to cope with uncorrelated sources of uncertainty for the different elements of the plant as well as with correlated ones, the computation of the solution is said to have high computational cost. Only the maximum value of the magnitude of elements of the RGA is calculated at one point in frequency domain for each computation. This is a difference with Dan Chen and Dale E. Seborg approach, where upper and lower bounds of the magnitude of the RGA are proposed.

In this paper a geometrical interpretation of the effect of model uncertainties on RGA elements is used to derive an uncertainty region for the RGA elements over frequency. Upper and lower bounds are computed from a symmetrical uncertainty region around the nominal model. The method is then applied to an explanatory example and a case study where a coal injection process for blast furnaces is analyzed.

\section{INTRODUCTION TO RGA}

The RGA of a square, complex, non-singular nxn matrix $A$ is defined as

$$
R G A(A) \triangleq A \otimes A^{-T}
$$

where $A^{-T}$ is the transpose of the inverse of $\mathrm{A}$, and $\otimes$ is the Schur multiplier, and denotes element by element multiplication.

The RGA was first introduced in [1] as an static interaction measure, built upon DC-gains. The RGA is mainly used for the analysis of interactions in multivariable processes, aiding the designer to select the proper pairing of inputs and outputs when decentralized control is possible. The pairing rules of the RGA can be summarized as:

- values of the RGA close to 1 are preferred for pairing,

- large values of the RGA are related to ill conditioned plants and should be avoided,

- negative values of the RGA must be avoided due to stability issues. 
The RGA has also been proven to be a useful tool for the analysis of interactions in frequency domain; the frequency dependant RGA was first introduced in [9], and is sometimes named as DRGA. This dynamic extension of the RGA is straightforward, and is the object of analysis in this paper.

The RGA of a 2-by-2 process $G_{2 x 2}(s)$

$$
G(s)=\left(\begin{array}{ll}
G_{11}(s) & G_{12}(s) \\
G_{21}(s) & G_{22}(s)
\end{array}\right)
$$

can be computed as

$$
\boldsymbol{R G A}(\mathbf{G}(\mathbf{s}))=\left(\begin{array}{cc}
\lambda_{11} & 1-\lambda_{11} \\
1-\lambda_{11} & \lambda_{11}
\end{array}\right)
$$

where

$$
\lambda_{11}=\frac{1}{1-\frac{G_{12}(s) G_{21}(s)}{G_{11}(s) G_{22}(s)}}
$$

The element $k=\frac{G_{12}(s) G_{21}(s)}{G_{11}(s) G_{22}(s)}$ has been referred as the interaction quotient in [10], and has been used in [7] to obtain the worst-case bounds of the static RGA. This so called interaction quotient is the basis of the analysis proposed in this paper.

\section{A. RGA and Model Uncertainty}

Assume we have a 2-by-2 MIMO system with nominal plant $G(s)$ as described in (1) and element by element multiplicative uncertainty of the form

$$
\Pi: G p(s)=G(s) \otimes(I+W(s))
$$

with

$$
W(s)=\left(\begin{array}{ll}
W_{11}(s) \cdot \Delta_{11}(s) & W_{12}(s) \cdot \Delta_{12}(s) \\
W_{21}(s) \cdot \Delta_{21}(s) & W_{22}(s) \cdot \Delta_{22}(s)
\end{array}\right)
$$

where $\Delta_{i j}(s), i, j=\{1,2\}$ is any stable transfer function with $\left|\Delta_{i j}\right| \leqslant 1$. This represents multiplicative uncertainty in each of the elements of the form

$$
G_{p_{i j}}=G_{i j}(s)\left(1+W_{i j}(s) \cdot \Delta_{i j}(s)\right), \forall i, j=\{1,2\}
$$

In this section the term $s$ will be dropped for the sake of simplicity. $G_{p}$ represents any particular perturbed plant, and $\Pi$ is the uncertainty set, which includes all the possible $G_{p}$. This representation assumes that the different sources of uncertainty $\Delta_{i j}$ are uncorrelated. It has also been used in [11] for the analysis of the RGA for uncertain systems. The advantage of multiplicative uncertainty is it's versatility. Most of the different representations are easily translatable into element by element multiplicative uncertainty by the proper selection of $W_{i j}$. The weakness of this representation lies in it's inability to represent the coupling in the sources of interaction, since the different $\Delta_{i j}$ are assumed to be independent. This usually gives a more conservative representation of the uncertainties when other descriptions like unstructured or structured uncertainty are translated into this one.

Now, the element $\lambda_{11}$ of the RGA for any perturbed plant $G_{p}$ can be given as:

$$
\lambda_{p 11}=\frac{1}{1-\frac{G_{12} \cdot G_{21}}{G_{11} \cdot G_{22}} \cdot \frac{\left(1+W_{12} \Delta_{12}\right)\left(1+W_{21} \Delta_{21}\right)}{\left(1+W_{11} \Delta_{11}\right)\left(1+W_{22} \Delta_{22}\right)}}
$$

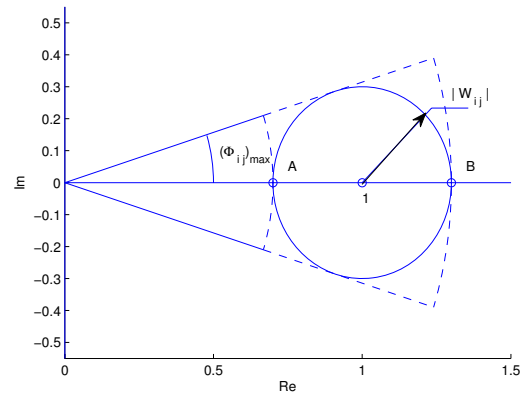

Fig. 1. Area representing all the possible values of $\left(1+W_{i j} \Delta_{i j}\right)$ at a given frequency $\omega$.

In this case, the interaction quotient will be factorized in two terms; one denoted as $G_{c}=\left(G_{12} G_{21}\right) /\left(G_{11} G_{22}\right)$ which can be referred to as nominal interaction quotient, and which is only depending on the nominal plant, and a second one denoted as $W_{c}=\left(\left(1+W_{12} \Delta_{12}\right)\left(1+W_{21} \Delta_{21}\right)\right) /((1+$ $\left.\left.W_{11} \Delta_{11}\right)\left(1+W_{22} \Delta_{22}\right)\right)$ which only depends on the uncertainties. $\lambda_{p_{11}}$ can then be expressed as

$$
\lambda_{p_{11}}=1 /\left(1-G_{c} \cdot W_{c}\right)
$$

The first step in the analysis will be then to obtain all the possible values of $W_{c}$.

\section{B. Bounds for $W_{c}$}

First the bounds of each of the terms $\left(1+W_{i j} \Delta_{i j}\right) ; \forall i, j=$ $\{1,2\}$ are derived.

Sections II-B and II-C refer to a given frequency $\omega$, and in the sequel, the term $(j \omega)$ will be dropped for the sake of simplicity.

At any given frequency $\omega,\left(1+W_{i j} \Delta_{i j}\right)$ represents a discshaped region of radius $\left|W_{i j}\right|$ centered in 1 .

Inspecting Fig. 1 the maximum and minimum values of the magnitude and phase of $\left(1+W_{i j} \Delta_{i j}\right)$ can be easily identified as corresponding to the points $\mathrm{A}$ and $\mathrm{B}$ respectively. Thus:

$$
\begin{aligned}
& \left|\left(1+W_{i j} \Delta_{i j}\right)\right|_{\text {min }}=\left(1-\left|W_{i j}\right|\right) \\
& \left|\left(1+W_{i j} \Delta_{i j}\right)\right|_{\text {max }}=\left(1+\left|W_{i j}\right|\right)
\end{aligned}
$$

Obviously, the phase of $\left(1+W_{i j} \Delta_{i j}\right)$ is bounded by $[-$ $\left.\left(\Phi_{i j}\right)_{\max },\left(\Phi_{i j}\right)_{\max }\right]$, where $\left(\Phi_{i j}\right)_{\max }=\operatorname{asin}\left(\left|W_{i j}\right|\right)$ The conclusion is that, $\left(1+W_{i j} \Delta_{i j}\right)$ at a certain frequency can be bounded as a complex region with magnitude included in the interval $\left[\left(1-\left|W_{i j}\right|\right),\left(1+\left|W_{i j}\right|\right)\right]$, and phase within $\left[-\operatorname{asin}\left(\left|W_{i j}\right|\right), \operatorname{asin}\left(\left|W_{i j}\right|\right)\right]$.

Once the possible values of each of the elements $(1+$ $\left.W_{i j} \Delta_{i j}\right)$ are known, a bound of $W_{c}$ can be generated at a given frequency. Assuming that $\left|W_{i j}\right| \leq 1 ; \quad \forall i, j=\{1,2\}$, the maximum and minimum values of $\left|W_{c}\right|$ can be computed as:

$$
\begin{aligned}
\left|W_{c}\right|_{\text {max }} & =\frac{\left(1+\left|W_{12}\right|\right)\left(1+\left|W_{21}\right|\right)}{\left(1-\left|W_{11}\right|\right)\left(1-\left|W_{22}\right|\right)} \\
\left|W_{c}\right|_{\text {min }} & =\frac{\left(1-\left|W_{12}\right|\right)\left(1-\left|W_{21}\right|\right)}{\left(1+\left|W_{11}\right|\right)\left(1+\left|W_{22}\right|\right)}
\end{aligned}
$$



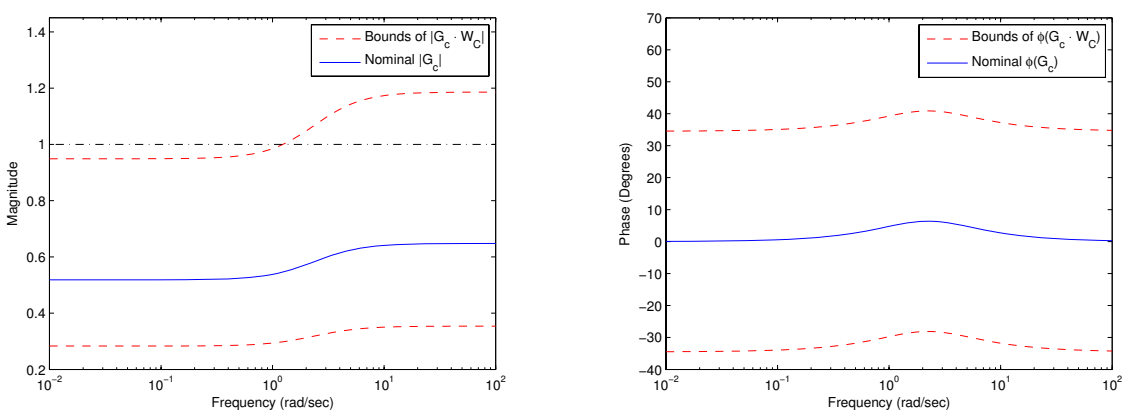

Fig. 2. Bounds of $\left|G_{c} \cdot W_{c}\right|$ (left) and $\Phi_{G_{c} W_{c}}$ (right) represented in frequency domain for the system described in (5).

The phase of $W_{c}$ is then included in the interval $\left[-\left(\Phi_{W_{c}}\right)_{\max },\left(\Phi_{W_{c}}\right)_{\max }\right]$, where $\left(\Phi_{W_{c}}\right)_{\max }$ is calculated as the sum of all the $\left(\Phi_{i j}\right)_{\max }$. That is:

$$
\left(\Phi_{W_{c}}\right)_{\max }=\sum_{i=1}^{2} \sum_{j=1}^{2} \operatorname{asin}\left(\left|W_{i j}\right|\right)
$$

\section{Bounds of $\lambda_{p_{11}}$}

Now that, the maximum and minimum values of $W_{c}$ at a given frequency $\omega$ are available, bounds for $\lambda_{p_{11}}$ can be derived. Representing $G_{c}$ and $W_{c}$ in polar form:

$$
\lambda_{p 11}=\frac{1}{1-\left(\left(\left|G_{c}\right| \angle \Phi_{g c}\right) \cdot\left(\left|W_{c}\right| \angle \Phi_{W_{c}}\right)\right)}
$$

at any given frequency $\omega, G_{c} \cdot W_{c}$ can then be bounded by:

$$
\begin{aligned}
\left|G_{c} \cdot W_{c}\right| & \in\left[\left|G_{c} \cdot W_{c}\right|_{\min },\left|G_{c} \cdot W_{c}\right|_{\max }\right] \\
\Phi_{G_{c} W_{c}} & \in\left[\left(\Phi_{G_{c} W_{c}}\right)_{\min },\left(\Phi_{G_{c} W_{c}}\right)_{\max }\right]
\end{aligned}
$$

where

$$
\begin{gathered}
\left|G_{c} \cdot W_{c}\right|_{\text {min }}=\left|G_{c}\right| \cdot\left|W_{c}\right|_{\text {min }} \\
\left|G_{c} \cdot W_{c}\right|_{\text {max }}=\left|G_{c}\right| \cdot\left|W_{c}\right|_{\text {max }} \\
\left(\Phi_{G_{c} W_{c}}\right)_{\text {min }}=\Phi_{G_{c}}-\left(\Phi_{W_{c}}\right)_{\text {max }} \\
\left(\Phi_{G_{c} W_{c}}\right)_{\text {max }}=\Phi_{G_{c}}+\left(\Phi_{W_{c}}\right)_{\max }
\end{gathered}
$$

\section{SingularitiES AND ROBUST INTEGRITY}

The nonsingularity of the plant is a necessary condition to asses the integrity of a system under decentralized control. It is also a necessary condition for the RGA to be bounded. The definition of integrity for nominal systems has been provided in [12]. A controlled system under decentralized control is said to achieve integrity if there exists a diagonal controller with integral action in each of the SISO controllers for which, the closed-loop system remains stable when the SISO controllers are brought in and out of service. In [8] integrity is defined for uncertain systems, also the following conditions for robust integrity are proposed as a generalization of the necessary and sufficient conditions derived in [13]. For an uncertain system, the system is said to achieve robust integrity if it achieves integrity for all the uncertainty set $\Pi$.

The necessary conditions for robust integrity are:

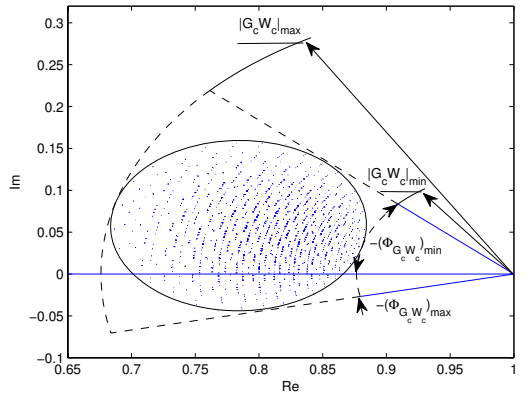

Fig. 3. Complex representation of the possible values of the element $\left(1-G_{c} W_{c}\right)$ at a given frequency $\omega$.

- $G_{p}$ is nonsingular.

- All the principal submatrices of $G_{p}$ are nonsingular.

Although they are an extension of the necessary and sufficient condition for nominal systems, these conditions have only been claimed to be necessary and not sufficient. In a 2-by-2 case, only the first condition is meaningful.

Inspecting (2) it can be observed that $\lambda_{p_{11}}$ becomes singular for values of $G_{c} \cdot W_{c}$ equal to 1 . That is, when $\left|G_{c} \cdot W_{c}\right|=1$ and $\Phi_{G_{c} W_{c}}=0$ simultaneously. In Fig. 2 the identified bounds of $\left|G_{c} \cdot W_{c}\right|$ and $\Phi_{G_{c} W_{c}}$ for the uncertain model described by (5) are depicted. This model has a constant value of $W_{i j}=0.15 \quad \forall i, j=\{1,2\}$.

$$
G(s)=\left(\begin{array}{cc}
\frac{5}{0.5 s+1} & \frac{2.4}{0.25 s+1} \\
\frac{2.7}{0.4 s+1} & \frac{2.5}{0.25 s+1}
\end{array}\right)
$$

The frequencies where $\left|G_{c} \cdot W_{c}\right|_{\min } \leq 1 \leq\left|G_{c} \cdot W_{c}\right|_{\max }$ and $\left(\Phi_{G_{c} W_{c}}\right)_{\min } \leq 0 \leq\left(\Phi_{G_{c} W_{c}}\right)_{\max }$ simultaneously, can be easily identified. At these frequencies, there might exist a combination of the inputs which make the plant become singular. If at a given frequency $\omega, 1 \notin\left[\left|G_{c} \cdot W_{c}\right|_{\text {min }}, \mid G_{c}\right.$. $\left.\left.W_{c}\right|_{\max }\right]$, or $0 \notin\left[\left(\Phi_{G_{c} W_{c}}\right)_{\min },\left(\Phi_{G_{c} W_{c}}\right)_{\max }\right]$, then the plant remains nonsingular for all possible $G_{p}$. This condition is similar to the one obtained in [11] for a description in which all the $W_{i j}$ are assumed to be equal.

\section{BOUNDS OF $\lambda_{p_{11}}$ AT A GIVEN FREQUENCY $\omega$}

Recall from Fig. 1 how each of the elements $\left(1+W_{i j} \Delta_{i j}\right)$ has been bounded by a complex number with magnitude inside the interval $\left[\left(1-\left|W_{i j}\right|\right),\left(1+\left|W_{i j}\right|\right)\right]$, and phase 


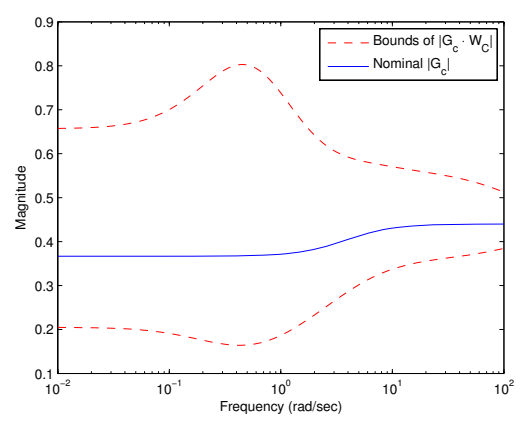

Fig. 4. Bounds of $\left|G_{c} \cdot W_{c}\right|$ represented in frequency domain for the system described in (7) and (8).

within $\left[-a \sin \left(\left|W_{i j}\right|\right), a \sin \left(\left|W_{i j}\right|\right)\right]$. Note that, both conditions together describe the area enclosed by the dashed lines, instead the disc-shaped region described by $\left(1+W_{i j} \Delta_{i j}\right)$. This means that the represented area has been overestimated. Instead, the following bound for the values of $W_{c}$ is then proposed:

$$
\begin{gathered}
\mathscr{C} \triangleq\left(\left|W_{c}\right|_{\max }+\left(c_{1} \cdot r_{2}+c_{2} \cdot r_{1}\right) \cdot(\cos (\theta)-1)\right. \\
+r_{1} \cdot r_{2} \cdot(\cos (2 \theta)-1), \\
\left.\left(c_{1} \cdot r_{2}+c_{2} \cdot r_{1}\right) \cdot \sin (\theta)+r_{1} \cdot r_{2} \cdot \sin (2 \theta)\right) \\
\theta \in[0,2 \pi]
\end{gathered}
$$

where

$$
\begin{gathered}
c_{1}=\left(1-\left|W_{11}\right|^{2}\right)^{-1} \quad ; \quad c_{2}=\left(1-\left|W_{22}\right|^{2}\right)^{-1} \\
r_{1}=\left(\left|W_{c}\right|_{\text {max }}-\left|W_{c}\right|_{\text {min }}-2 \cdot c_{1} \cdot r_{2}\right) / 2 \cdot c_{2} \\
r_{2}=\max _{i, j}\left(\left\{\left|W_{i j}\right|\right\}\right) \cdot c_{2}
\end{gathered}
$$

At a given frequency $\omega, \mathscr{C}$ represents an approximation of the curve enclosing all the possible vales of $W_{c}$ in cartesian coordinates. And it can be applied at the frequencies where $\left|W_{i j}\right| \leq 1 ; \forall i, j=\{1,2\}$. In Fig. 3 the created bound can be observed, and it's behavior representing the possible values of $\left(1-G_{c} W_{c}\right)$, it is also compared with the previously overestimated bound described by conditions (3) and (4), which clearly define the area enclosed by the dashed line. The inverse of the obtained area will represent the possible values of the element $\lambda_{p_{11}}$. Note that if that area encloses the point $(0,0)$, the plant might then become singular in the uncertainty set at the analyzed frequency. Now an uncertainty region can be given for a nominal RGA at each analyzed frequency. The algorithms are implemented in Matlab and will be discussed with the following examples.

\section{EXAMPLE}

The RGA of the uncertain system described by (7) and (8) will be now analyzed.

$$
\begin{gathered}
G(s)=\left(\begin{array}{cc}
\frac{5}{0.4 s+1} & \frac{2.75}{0.25 s+1} \\
\frac{-2}{0.4 s+1} & \frac{3}{0.3 s+1}
\end{array}\right) \\
W(s)=\left(\begin{array}{cc}
\frac{0.25}{s+1} & \frac{s+0.06667}{3 s^{2}+4 s+1} \\
\frac{0.24}{2 s+1} & \frac{0.3832 s+0.01533}{0.02 s^{2}+2.01 s+1}
\end{array}\right)
\end{gathered}
$$

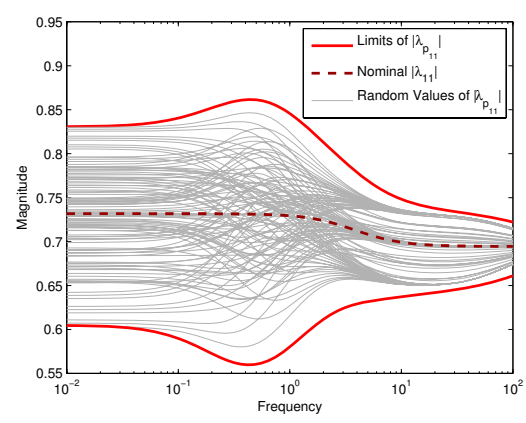

Fig. 5. Bounds of the element $\lambda_{p_{11}}$ for the system described in (7) and (8).

Clearly from Fig. 4, the plant remains nonsingular at the analyzed frequencies, and the RGA is bounded.

The maximum and minimum values of the magnitude of the RGA are then generated at different frequencies from the bounds defined in (6), and depicted in Fig. 5. In this example the uncertainties described by $W_{i j}$ are low. Each of the values of $\left|W_{i j}\right|$ are lower than 0.25 at any frequency. The nominal RGA suggests diagonal pairing, but it can be seen how these values are easily perturbed to values which mean high interaction between channels and invalidate the selection for decentralized control design.

This example shows how values of the nominal RGA which are not close to 1 or 0 , can be easily perturbed. An analysis of the RGA to uncertainties could then be done to asses or reject the decision taken from the nominal plant. Evidence suggests that for higher order systems, the sensitivity of the RGA will get worse.

\section{ReAl LifE EXAMPLE}

\section{A. Coal injection vessel}

The coal injection vessel is a pressurized multivariable tank system which is discussed in [14], [15] and [16]. There, models for the process are derived and successfully used for the design and analysis of multivariable controllers and a gas-leakage detection system.

The process can be described shortly as follows. During the injection phase of the vessel, the pressure control valve $u_{N}$ and the flow control valve $u_{C}$ are used to release a constant coal flow from the vessel. The coal flow cannot be measured directly. The available measurements are the net weight $m_{T}$ of the vessel, which is the sum of the nitrogen weight $m_{N}$ and the fine coal weight $m_{C}$, and the pressure in the vessel $p$. A linear physical model for the coal injection vessel for the injection phase is given by

$$
\begin{aligned}
\dot{x}(t)= & 10^{-3}\left[\begin{array}{rr}
-0.3234 & 0.3604 \\
0.2128 & -0.2963
\end{array}\right] x(t) \\
& +\left[\begin{array}{rr}
-0.4878 & 0.6816 \\
0.5721 & -0.3043
\end{array}\right] u(t) \\
y(t)= & {\left[\begin{array}{rr}
-0.8379 & 0.6477 \\
-0.0237 & -0.0198
\end{array}\right] x(t) }
\end{aligned}
$$



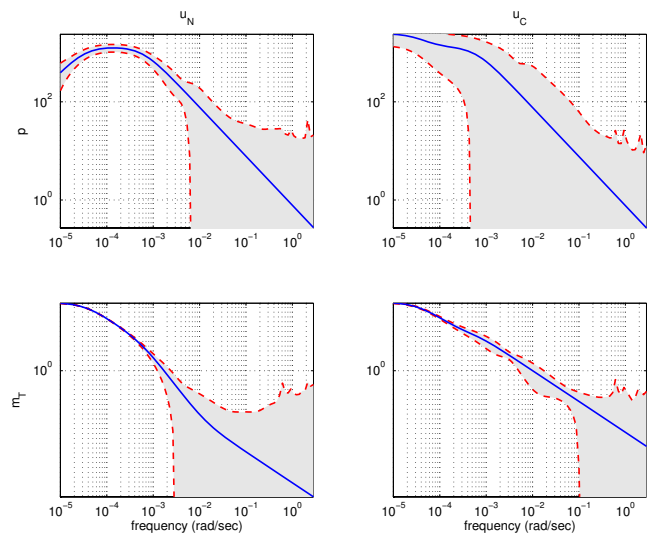

Fig. 6. Bode magnitude plot for the nominal process $G_{P C I}$ (solid) with uncertainty regions (shaded) with upper and lower bounds (dashed).

where $u(t)=\left[u_{N}(t) u_{C}(t)\right]^{T}$ and $y(t)=\left[p(t) m_{T}(t)\right]^{T}$. In the sequel, individual transfer operators are referred to as $G_{P C I_{i j}}$ and the complete model as $G_{P C I}$.

\section{B. Error modeling}

In order to analyze the effect of uncertainties on the RGA analysis it is necessary to derive a description for the actual uncertainty. For this end logged data from the process is used in order to perform model error modeling (MEM). In accordance with [17], a high order model $G_{e}$ relating the logged input with for the residual output of the linear model $G_{P C I}$ is derived with the prediction error method. Model validation is performed using fresh secondary data sets.

The uncertainty regions are then derived in the frequency domain by constructing a symmetric region around the nominal process model $G_{P C I}$. First, an uncertainty region around $G_{e}+G_{P C I}$ is computed from the $99.9 \%$ confidence interval. Then, this region is expanded in the upper and lower magnitude to achieve a symmetric region around $G_{P C I}$. The resulting uncertainty regions are depicted in Fig. 6.

Clearly, the uncertainty region is expanding for higher frequencies. Additionally, the transfer function relating to the vessel pressure have a larger uncertainty region. A reason for this can be found in the assumptions and simplifications that were applied during the modeling, as pressure in the nitrogen net and fluidization flows in the lower part of the vessels were ignored. These process variables vary during an injection cycle and cause disturbances which can be translated into gain changes and leakages in the valve $u_{N}$, respectively.

\section{Discussion}

There are three different frequency regions that are of interest.

- Low frequencies. Below $10^{-5} \mathrm{rad} / \mathrm{sec}$.

- Frequencies between $10^{-5} \mathrm{rad} / \mathrm{sec}$ to $10^{-3.3} \mathrm{rad} / \mathrm{sec}$.

- High frequencies. Above $10^{-3.3} \mathrm{rad} / \mathrm{sec}$.

Below $10^{-5} \mathrm{rad} / \mathrm{sec}$ the element $\lambda_{p_{11}}$ is close to 0 , suggesting off-diagonal pairing. The gain of the transfer function $G_{P C I_{11}}$ is very uncertain, which leads to large values of $W_{11}$ in order to represent all the uncertainty

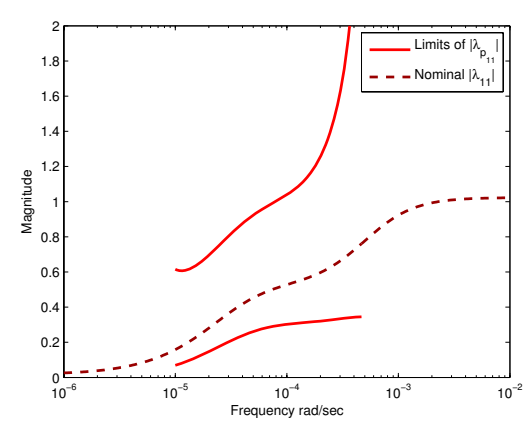

Fig. 7. Element $\lambda_{p_{11}}$ on the RGA of the Vessel.

set. The result is that $W_{11}$ exceeds the value 1 ; and the assumption $\left|W_{i j}(j \omega)\right| \leq 1 ; \quad \forall i, j=\{1,2\}$ made in II$\mathrm{B}$ is not satisfied. The rest of the elements $W_{i j}$ remain lower than 1. At frequencies below $10^{-6} \mathrm{rad} / \mathrm{sec}$ the nominal magnitude of $\lambda_{p_{11}}$ is close to 0.02 , values close to 0 (or 1 ) imply robustness to uncertainties, so the nominal value can usually be trusted, unless very large uncertainties are present. In this case, a very loose upper limit to the magnitude of $\lambda_{p_{11}}$ can be generated in the following way

$\lambda_{p_{11}}=a b s\left(\frac{1}{1-\frac{\min \left(\left|G_{P C I_{12}}\right|\right) \cdot \min \left(\left|G_{P C I_{21}}\right|\right)}{\max \left(\left|G_{P C I_{11}}\right|\right) \cdot \max \left(\left|G_{P C I_{22}}\right|\right)}}\right)=0.26$

which still can be used for pairing purposes. Nevertheless, from process knowledge, we know that an injection cycle lasts for less than 2000 seconds which means that very low frequencies are not of interest for control actions.

The second region includes frequencies between $10^{-5} \mathrm{rad} / \mathrm{sec}$ to $10^{-3.3} \mathrm{rad} / \mathrm{sec}$. Fig. 8 represents the possible values of the magnitude and phase of $G_{c} \cdot W_{c}$ at the frequencies where $\left|W_{i j}(j \omega)\right| \leq 1 ; \quad \forall \omega ; \quad i, j=\{1,2\}$. It can be observed at the represented frequencies above $10^{-4.62} \mathrm{rad} / \mathrm{sec}$ that possible values of $\left|G_{c} \cdot W_{c}\right|$ include 1 , but 0 is not one of the possible values of $\Phi\left(G_{c} \cdot W_{c}\right)$. Then, at these frequencies the plant remains nonsingular, and the RGA bounded for all the uncertainty set. In Fig. 7 the bounds of the element $\lambda_{p_{11}}$ are depicted for this range of frequencies using the algorithm described in IV. Decisions about pairing for decentralized control cannot be adopted if this bounds are taken into consideration.

At frequencies above $10^{-3.3} \mathrm{rad} / \mathrm{sec}$ the uncertainty set becomes larger and the assumption $\left|W_{i j}(j \omega)\right| \leq 1 ; \quad \forall i, j=$ $\{1,2\}$ is not satisfied again. The plant $G_{P C I}$ is close to upper triangularity, and the RGA becomes close to identity, suggesting diagonal pairing. In this case, the RGA suggests the correct pairing for decentralized control, but fails to produce any information about the coupling between the different channels. In this case the analysis of the RGA to uncertainties is not of interest, since only perturbations which can make the plant divert from triangularity can perturb the RGA in a noticeable way, and this case is not possible in the uncertainty set. 

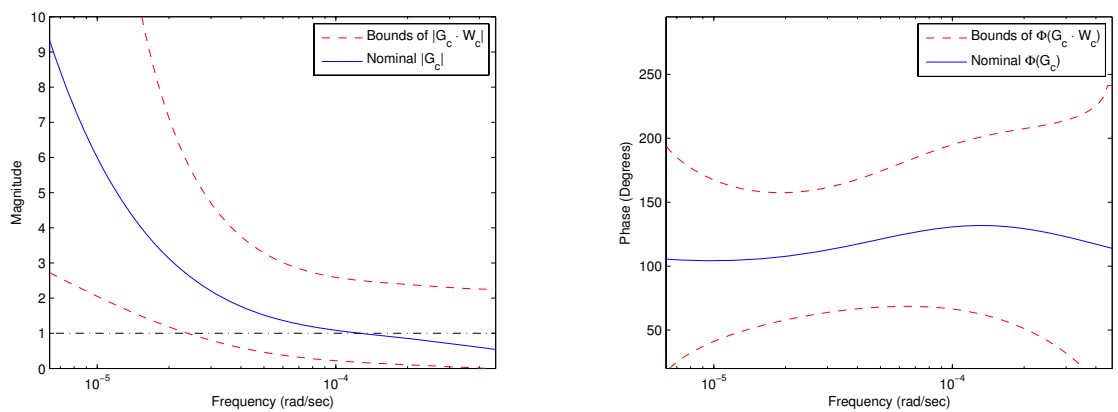

Fig. 8. Bounds of $\left|G_{c} \cdot W_{c}\right|$ (left) and $\Phi_{G_{c} W_{c}}$ (right) represented in frequency domain.

\section{CONCLUSION}

Correct decisions in control structure design depend on the quality of the process models the decisions are based upon. Hence, uncertainties in a process model affect the validity of decisions based on the RGA analysis. Therefore uncertainty regions of the RGA that are computed from uncertainty regions of a process model are needed. For the 2-by-2 case a methodology is derived that produces tight bounds for RGA elements. These bounds support engineers to assess the validity of their decisions and if it is possible to make decision based on the RGA at all. Clearly, if an RGA element has a large uncertainty region, it is questionable to base a decision on the nominal value. Still, the method is preliminary in the sense that it only addresses the 2-by2 case. It has to be generalized to n-by-n cases and its performance has to be compared with the methods proposed in [7] and [8].

From the analyzed cases it can be concluded that only values of the RGA close to 1 or 0 , are robust enough to multiplicative uncertainty, so a decision on the structure selection can be made without a previous analysis to uncertainties. Nevertheless, large uncertainties can invalidate the decisions taken on the nominal case.

From the model analyzed in Section $\mathrm{V}$ it can be concluded that values of the nominal RGA which differ from 0 , or 1 , can be easily perturbed by uncertainties. A real life example was then analyzed in Section VI to asses the validity of the conclusions. For a real process like the one analyzed uncertainties are observed to be even much larger, and the RGA is clearly perturbed from the nominal values.

Multiplicative uncertainty can only represent symmetric deviations from the nominal plant, and also the different sources of uncertainty are assumed to be uncorrelated. This leads to more conservative representation of the uncertainties. This paper deals with the worst case bounds due to multiplicative uncertainty. Thus, an analysis of how likely a process is perturbed in such a way that the RGA becomes largely uncertain is needed.

\section{REFERENCES}

[1] E. Bristol, "On a new measure of interaction for multi-variable process control," IEEE Transactions on Automatic Control, vol. AC-11, no. 1, pp. 133 - 134, 1966/01/.
[2] K. E. Häggblom, "Control structure analysis by partial relative gains," in Proc. of the 36th Conference on Decision \& Control, San Diego, USA, December 1997, pp. 2623-2624.

[3] P. Grosdidier and M. Morari, "The $\mu$ interaction measure," Ind. Eng. Chem Res., vol. 26, pp. 1193-1202, 1987.

[4] M. E. Salgado and A. Conley, "Mimo interaction measure and controller structure selection," Internation Journal of Control, vol. 77, no. 4, pp. 367-383, 2004.

[5] B. Wittenmark and M. E. Salgado, "Hankel-norm based interaction measure for input-output pairing," in Proc. of the 2002 IFAC World Congress, Barcelona, 2002

[6] W. Birk and A. Medvedev, "A note on gramian-based interaction measures," in Proc. of the European Control Conference 2003, University of Cambridge, UK, 2003.

[7] D. E. S. Dan Chen, "Relative gain array analysis for uncertain process models," AIChE Journal, vol. 48, no. 2, pp. 302-310, 2002. [Online]. Available: http://dx.doi.org/10.1002/aic.690480214

[8] V. Kariwala, S. Skogestad, and J. Forbes, "Relative gain array for norm-bounded uncertain systems," Industrial \& Engineering Chemistry Research, vol. 45, no. 5, pp. 1751-1757, 2006. [Online]. Available: http://pubs3.acs.org/acs/journals/doilookup?in oi i $=$ $10.1021 / i e 050790 r$

[9] M. Witcher and T. McAvoy, "Interacting control systems: steady state and dynamic measurement of interaction," ISA Transactions, vol. 16, no. 3, pp. $35-41,1977 / /$, multivariable control system;relative gain array;interaction measure;dynamic interactions;interacting control loops;interaction measurement;.

[10] J. Rijnsdorp, "Interaction in two-variable control systems for distillation columns - i and ii," Automatica, vol. 1,15, 1965.

[11] S. Skogestad and M. Morari, "Implications of large rga-elements on control performance," Industrial and Engineering Chemistry Research, vol. 26, no. 11, pp. 2323-2330, 1987. [Online]. Available: http : //pubs3.acs.org/acs/journals/doilookup? $i n_{d}$ oi $=$ 10.1021/ie00071a025

[12] M. Campo, P.J.; Morari, "Achievable closed-loop properties of systems under decentralized control: conditions involving the steady-state gain," Automatic Control, IEEE Transactions on, vol. 39, no. 5, pp. 932-943, May 1994.

[13] V. Kariwala, J. F. Forbes, and E. S. Meadows, "Integrity of systems under decentralized integral control." [Online]. Available: citeseer.ist.psu.edu/kariwala05integrity.html

[14] A. Johansson and A. Medvedev, "Model based leakage detection in a pulverized coal injection vessel," IEEE Transactions on Control Systems Technology, vol. 7, no. 6, pp. 675-682, November 1999.

[15] W. Birk and A. Medvedev, "Sensitivity analysis of an LQ optimal multivariable controller for a fine coal injection vessel," IEEE Transactions on Industry Applications, vol. 36, no. 3, pp. 871-876, May/June 2000.

[16] _ - "Pressure and flow control of a pulverized coal injection vessel," IEEE Transactions on Control Systems Technology, vol. 8, no. 6, pp. 919-929, November 2000

[17] W. Reinelt, A. Garulli, and L. Ljung, "Comparing different approaches to model error modelling in robust identification," Automatica, vol. 38 , no. 5, pp. 787-803, 2002. 